\title{
Théorème de Bayes et rapports de vraisemblance
}

\author{
M.R. Nendaz, A. Perrier
}

Clinique de Médecine 1, Hôpitaux Universitaires de Genève, Suisse.

Tirés à part : M. Nendaz, Clinique de Médecine 1,

Hôpitaux Universitaires de Genève, Rue Micheli-du-Crest 24,

$\mathrm{CH}-1211$ Genève 14, Suisse.

mathieu.nendaz@hcuge.ch

Réception version princeps à la Revue : 24.09.2003.

Retour aux auteurs pour révision : 31.10.2003.

Réception $1^{\text {ère }}$ version révisée : 24.11.2003.

Acceptation définitive : 26.11.2003.

Nous avons vu dans le mémento précédent [1] que les notions de sensibilité et spécificité décrivent les propriétés intrinsèques d'un test, et que la valeur prédictive positive (VPP) et la valeur prédictive négative (VPN) indiquent la probabilité de porter, ou non, la maladie recherchée par le test, en fonction de son résultat positif ou négatif. Appliquons cela en pratique en utilisant l'exemple de l'utilité du dosage du Peptide Natriurétique de type B (PNB) pour diagnostiquer une insuffisance cardiaque face à une dyspnée aiguë [2]. Rappelons que dans cette étude multicentrique effectuée en milieu d'urgence, 1538 patients avec dyspnée aiguë ont été inclus, dont 722 (prévalence de $47 \%$ ) ont été finalement considérés comme souffrant réellement d'insuffisance cardiaque par deux cardiologues de référence n'ayant pas connaissance de la valeur du dosage du PNB. Une valeur de PNB à $100 \mathrm{pg} / \mathrm{ml}$ ou plus était considérée comme positive pour une insuffisance cardiaque, et les caractéristiques de ce test sont résumées dans le tableau $I$.

\section{Valeurs prédictives et prévalence de la maladie recherchée}

Dans notre exemple, la prévalence d'insuffisance cardiaque est de $47 \%$ dans cette population de patients. Cette pré-

Tableau I.

Caractéristiques du dosage du Peptide Natriurétique de type B (PNB) pour diagnostiquer une insuffisance cardiaque face à une dyspnée aiguë, (une valeur-seuil de $100 \mathrm{pg} / \mathrm{ml}$ ou plus définissant un test positif) [2]. La prévalence d'insuffisance cardiaque est de $47 \%$ dans cette étude.

\begin{tabular}{lccc}
\hline & $\begin{array}{c}\text { IC réellement } \\
\text { présente }\end{array}$ & $\begin{array}{c}\text { IC réellement } \\
\text { absente }\end{array}$ & Total \\
\hline PNB + & 650 & 220 & 870 \\
PNB - & 72 & 596 & 668 \\
Total & $722^{*}$ & 816 & $1538^{*}$ \\
\hline
\end{tabular}

*Données disponibles dans l'étude de McCullough [2], les autres valeurs étant déduites à partir des caractéristiques du test diagnostique mentionnées dans l'étude. Prévalence $=722 / 1538=47 \%$. Sensibilité $=650 / 722=90 \%$. Spécificité $=596 / 816=73 \%$. VPP $=650 / 870=75 \%$. VPN $=596 / 668=89 \%$. 
valence représente donc la probabilité initiale qu'un patient de cette population ait une insuffisance cardiaque, avant que l'on n'effectue un quelconque autre test. On l'appelle également probabilité a priori ou probabilité pré-test.

La valeur prédictive positive, comme nous l'avons vu dans le mémento précédent [1], est la probabilité de souffrir d'insuffisance cardiaque si le PNB est positif. La valeur prédictive négative est quant à elle la probabilité de ne pas présenter d'insuffisance cardiaque quand le PNB est négatif. La probabilité de souffrir d'une insuffisance cardiaque en cas de test négatif devient donc 1-VPN. La probabilité de souffrir de la maladie recherchée selon que le test est positif ou négatif est également nommée probabilité a posteriori ou probabilité post-test. Elle est respectivement de $75 \%$ si le PNB est positif et de $11 \%(100 \%$ moins $89 \%)$ si le PNB est négatif (tableau I).

Dans cet exemple, nous voyons donc que si la probabilité pré-test d'insuffisance cardiaque est de $47 \%$, la probabilité post-test monte à $75 \%$ si le $\mathrm{PNB}$ est positif, mais tombe à $11 \%$ si le PNB est négatif. Que se passe-t-il lorsque la probabilité pré-test est différente?

Admettons que nous appliquions le dosage du PNB à un patient dont la probabilité a priori d'insuffisance cardiaque est faible, par exemple $10 \%$. Les caractéristiques intrinsèques du test, à savoir sa sensibilité et sa spécificité restant les mêmes, on peut construire le tableau II. Nous remarquons que la valeur prédictive positive diminue à $27 \%$ alors que la valeur prédictive négative est plus élevée (98\%). La probabilité a posteriori de souffrir d'insuffisance cardiaque est donc de $27 \%$ si le PNB est positif et de $2 \%(100 \%-98 \%)$ si le test est négatif. Nous réalisons ainsi que, alors que les caractéristiques intrinsèques du test restent les mêmes (sensibilité de $90 \%$ et spécificité de $73 \%$ ), les valeurs prédictives, et donc la probabilité a posteriori de souffrir de la maladie recherchée selon le résultat du test, varient en fonction du groupe à qui il est appliqué, c'est-à-dire en fonction de la probabilité a priori (prévalence) de cette maladie.

\section{Tableau II.}

Caractéristiques du dosage du Peptide Natriurétique de type B (PNB) pour diagnostiquer une insuffisance cardiaque face à une dyspnée aiguë, (une valeur-seuil de $100 \mathrm{pg} / \mathrm{ml}$ ou plus définissant un test positif) [2]. La prévalence d'insuffisance cardiaque est de $10 \%$ dans cet exemple.

\begin{tabular}{lccc}
\hline & $\begin{array}{c}\text { IC réellement } \\
\text { présente }\end{array}$ & $\begin{array}{c}\text { IC réellement } \\
\text { absente }\end{array}$ & Total \\
\hline PNB + & 139 & 374 & 512 \\
PNB - & 15 & 1010 & 1026 \\
Total & 154 & 1384 & 1538 \\
\hline
\end{tabular}

Prévalence $=154 / 1538=10 \%$. Sensibilité $=139 / 154=90 \%$. Spécificité $=$ $1010 / 1384=73 \%$. VPP $=139 / 512=27 \%$. VPN $=1010 / 1026=98 \%$.

\section{Établissement de la probabilité post-test : le théorème de Bayes}

Nous avons vu que nous pouvons déterminer la probabilité post-test d'une maladie en calculant les valeurs prédictives positive et négative, et que ces dernières varient en fonction de la probabilité pré-test (ou prévalence) de la maladie. En pratique, lorsqu'on effectue un test, on ne connaît en général que sa sensibilité et sa spécificité, ce qui rend fastidieux la construction des classiques tableaux à double entrée (tels les tableaux I et II) contenant les nombres absolus de patients dans chaque cellule, puisqu'il faudrait utiliser une population fictive (par exemple de 1000 patients) pour calculer les valeurs prédictives. C'est ici qu'intervient le théorème du Révérend Bayes, un mathématicien du $18^{\mathrm{e}}$ siècle, qui permet de déterminer la probabilité a posteriori d'une maladie en fonction du résultat du test, à partir des seules valeurs de probabilité a priori, sensibilité et spécificité du test. L'examen des formules de Bayes nous montre qu'on retrouve les notions de valeurs prédictives faisant intervenir, non plus les nombres absolus de vrai et faux positifs ou de vrais et faux négatifs, mais directement les valeurs de sensibilité, spécificité, et probabilité pré-test. Selon ce théorème, si le test est positif, la probabilité post-test devient :

$\mathbf{P}$ post-test $=\mathbf{p}$ prétest ${ }^{*}$ sensibilité/ $\left(\mathbf{p}\right.$ prétest ${ }^{*}$ sensibilité $+(1-p \text { prétest })^{*}(1-$ specificité $\left.)\right)$

Si le test est négatif, la probabilité post-test devient :

$\mathbf{P}$ post-test $=\mathbf{p}$ prétest $^{*}(1-$ sensibilité $) /\left(\mathbf{p}\right.$ prétest ${ }^{*}$ (1-sensibilité) + (1-p pretest $)^{*}$ spécificité)

Ces calculs pouvant être ardus en milieu clinique, on peut s'aider de feuilles de calculs d'un tableur courant ou de logiciels conçus pour mini-ordinateurs de poche (www.medcalc.media.net).

En appliquant ce théorème à notre exemple de dosage du $\mathrm{PNB}$, on peut déterminer la probabilité d'insuffisance cardiaque en fonction de diverses valeurs de probabilité pré-test et représenter les résultats sous forme graphique (fig. 1). Cette figure illustre l'importance de l'influence de la probabilité a priori sur la probabilité a posteriori, alors que le même test, avec les mêmes caractéristiques (sensibilité, spécificité et rapport de vraisemblance) est utilisé. Ainsi, en prenant l'exemple du PNB sur la figure 1, si la probabilité pré-test de la maladie est très élevée (p. ex. $95 \%$ ), sa probabilité post-test restera encore relativement élevée malgré un test négatif (72\% en l'occurrence). Inversement, si la probabilité a priori de la maladie est très faible (p. ex. $5 \%$ ), sa probabilité a posteriori n'augmentera pas forcément de manière significative cliniquement, même si le test revient positif (en l'occurrence $15 \%$ ). Ceci illustre bien l'importance, avant d'effectuer un test, de déterminer la probabilité a priori de la maladie chez un patient particulier et d'anticiper ce que deviendra sa probabilité a posteriori en fonction du résultat du test. 


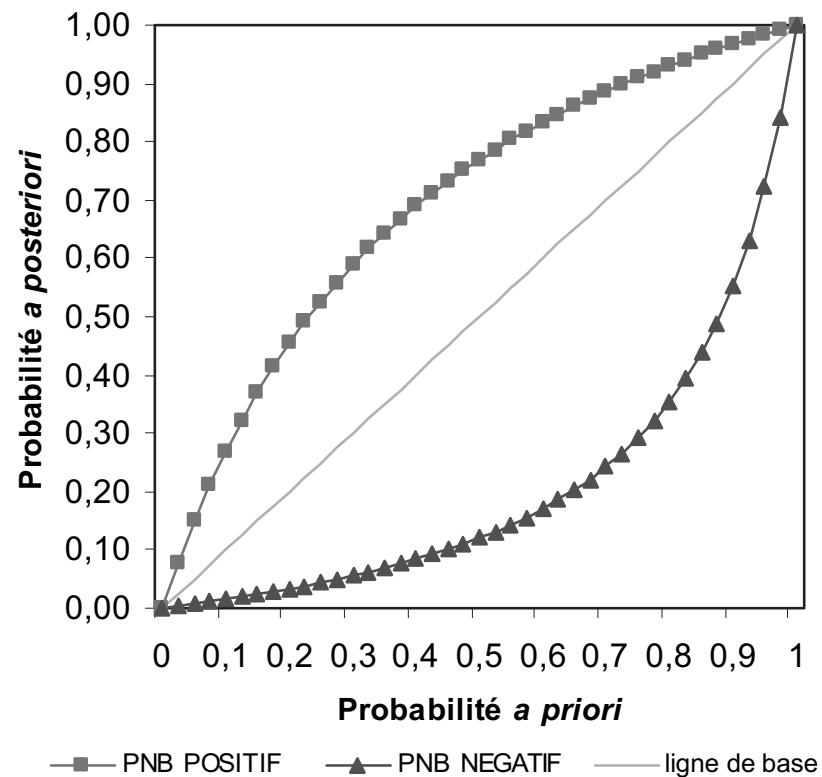

Fig. 1.

Application du théorème de Bayes : probabilité a posteriori d'insuffisance cardiaque en fonction de sa probabilité a priori et du résultat du dosage du PNB (une valeur-seuil de $100 \mathrm{pg} / \mathrm{ml}$ ou plus définissant un test positif).

Remarquons également que nous utilisons dans cet exemple un résultat binaire (positif ou négatif) d'un dosage qui fournit en réalité des valeurs continues. Ceci ne fait pas l'objet de ce mémento, mais le lecteur intéressé pourra constater en lisant l'article de McCullough et coll. [2] que ces auteurs ont également établi un modèle prédisant la probabilité post-test d'insuffisance cardiaque en fonction de la probabilité pré-test et de la valeur absolue de PNB.

\section{Établissement de la probabilité post-test : les rapports de vraisemblance}

A ce stade, vous avez en main toutes les notions nécessaires à l'utilisation pratique des caractéristiques d'un test, à savoir la détermination de la probabilité post-test à partir de la probabilité pré-test de la maladie recherchée. Pour être complet, il faut maintenant aborder, non pas des notions de base supplémentaires, mais une autre manière de les exprimer. Il s'agit des rapports de vraisemblance (RV) et des odds (ou « rapports de cote »), fréquemment utilisés dans certaines publications.

\section{Odds versus probabilités}

Un odds (cote) n'est qu'une des façons d'exprimer une probabilité, comme aux champs de course [3]. Il exprime au numérateur la probabilité qu'un événement se produise et au dénominateur la probabilité qu'il ne se produise pas. Ainsi, mathématiquement, odds $=\mathrm{p} /(1-\mathrm{p})$, et inversement, $\mathrm{p}=$ odds $(1+o d d s)$. Par exemple, si l'odds de souffrir d'une maladie est de $4: 1$, cela signifie que le risque de présenter la maladie est quatre fois plus élevé que celui de ne pas la présenter, ce qui représente une probabilité de $80 \%($ odds $/(1+$ odds $)=4 / 5=$ $0,8)$.

\section{Rapport de vraisemblance}

Le RV d'un test combine sa sensibilité et sa spécificité en un facteur unique et permet de simplifier le calcul de la probabilité post-test en utilisant les odds. Si le test est positif, le RV positif découle de la formule : fraction de vrais positifs/fraction de faux positifs, soit sensibilité/(1-spécificité). Si le test est négatif, le RV négatif est donné par: fraction de faux négatifs/fraction de vrais négatifs, soit (1-sensibilité)/spécificité.

Si le RV positif ou négatif d'un test est de 1 , cela signifie que l'odds initial de la maladie ne sera pas modifié par le test et que ce dernier n'aura aucune utilité pour confirmer ou exclure la maladie. Lorsque le RV est supérieur à 1 , l'odds de la maladie augmentera après le test, d'autant plus que ce rapport est élevé. Comme ordre de grandeur, un RV positif à 10 ou plus rendra le diagnostic très probable. S'il est inférieur à 1 , l'odds diminuera d'autant plus que cette valeur est faible : un RV à 0,1 ou moins va, en effet, quasiment exclure la maladie recherchée.

L'avantage de cette manière d'exprimer les notions de probabilité, sensibilité et spécificité réside dans le fait que les calculs du théorème de Bayes peuvent être grandement simplifiés, puisqu'on peut directement multiplier l'odds pré-test par le RV pour obtenir l'odds post-test (odds post-test = odds prétest*RV). Si plusieurs tests successifs sont conduits, on trouve l'odds final en multipliant simplement l'odds initial par les RV des différents tests effectués (odds final = odds initial ${ }^{*} \mathrm{RV} 1{ }^{*} \mathrm{RV} 2 * \mathrm{RV} 3{ }^{*}$...). Il ne reste ensuite qu'à reconvertir les odds en probabilités par un calcul simple pour se retrouver en terrain connu ! Notons qu'on peut également directement utiliser des probabilités avec des RV, sans faire le détour par les odds, en utilisant des nomogrammes publiés [4, 5], ou des logiciels pour ordinateurs de poche (www.medcalc.media.net).

Vérifions ceci en reprenant les données de notre exemple (tableau I). Transformons tout d'abord la probabilité initiale d'insuffisance cardiaque de $47 \%$ en odds. Nous trouvons un odds pré-test de $0,89(0,47 /(1-0,47))$. Calculons le RV d'un PNB positif : il est de $3,3(0,90 /(1-0,73))$. Si le test est positif, l'odds post-test devient $0,89 * 3,3$, soit 2,93 , ce qui donne bel et bien une probabilité post-test de $75 \%$, une fois l'odds reconverti $(2,93 /(1+2,93)$. Le RV d'un PNB négatif est quant à lui de $0,14((1-0,90) / 0,73)$. Si le test est négatif, l'odds post-test devient donc $0,12\left(0,89^{*} 0,14\right)$, ce qui correspond à une probabilité de $11 \%$, valeur superposable à celle trouvée en appliquant le théorème de Bayes. 


\section{Conclusion}

En résumé, alors que sensibilité et spécificité représentent les propriétés intrinsèques d'un test, la valeur prédictive d'un résultat positif ou négatif, c'est-à-dire la probabilité a posteriori d'avoir la maladie recherchée, varie selon la probabilité a priori. Le calcul de ce que devient la probabilité initiale de la maladie recherchée une fois le résultat du test connu fait appel au théorème de Bayes ou, alternativement, à l'utilisation des rapports de vraisemblance associés aux odds.

Au-delà des formules mathématiques, dont l'application est facilitée par divers logiciels de poche, la compréhension de ces notions devrait permettre au clinicien une lecture critique des publications concernant des tests diagnostiques [6]. En pratique, elles offrent également la possibilité d'anticiper l'effet du résultat d'un test sur la probabilité de la maladie recherchée, et donc d'en évaluer la pertinence clinique avant de l'effectuer.

\section{Références}

1 Nendaz M, Perrier A : Sensibilité, spécificité, valeur prédictive positive et valeur prédictive négative d'un test diagnostique. Rev Mal Respir $2004 ; 21: 390-3$.

2 McCullough PA, Nowak RM, McCord J, Hollander JE, Herrmann HC, Steg PG, Duc P, Westheim A, Omland T, Knudsen CW, Storrow AB, Abraham WT, Lamba S, Wu AH, Perez A, Clopton P, Krishnaswamy P, Kazanegra R, Maisel AS : B-type natriuretic peptide and clinical judgment in emergency diagnosis of heart failure: analysis from Breathing Not Properly (BNP) Multinational Study. Circulation 2002; $106: 416-22$.

3 Morabia A : Risques relatifs et odds ratio. Rev Mal Respir 2003 ; 20 : 757-9.

4 Fagan TJ : Nomogram for Bayes theorem. N Engl J Med $1975 ; 293$ : 257.

5 Sackett D, Haynes R, Guyatt G, Tugwell P : Clinical epidemiology. A basic science for clinical medicine. 2nd ed. Boston : Little, Brown and Co ; 1991.

6 Nendaz MR, Perrier A : Étude de validation d'un test diagnostique : un guide de lecture critique. À propos de la place de la biopsie endobronchique dans le diagnostic de la sarcoïdose. Rev Mal Respir 2002; 19 : 767-77. 